\title{
Segmental Orientation in Deformed Networks. 2. Molecular Theory for Biaxial Deformation
}

\author{
Zeynep Saraç, Burak Erman, and Ivet Bahar* \\ Polymer Research Center and School of Engineering, Bogazici University, and \\ TUBITAK Advanced Polymeric Materials Research Center, Bebek 80815, Istanbul, Turkey
}

Received July 20, 1994; Revised Manuscript Received October 5, $1994^{\otimes}$

\begin{abstract}
The Nagai formulation of segmental orientation in stretched polymer chains is applied to segmental orientation in amorphous elastomeric networks under biaxial deformation. The orientation of a reference vector, $\mathbf{m}$, rigidly affixed to a chain of the biaxially drawn network is investigated. The orientation of $\mathbf{m}$ is characterized by two orientation functions, $S_{x}$ and $S_{y}$, where $x$ and $y$ are the laboratoryfixed axes along which the macroscopic biaxial deformation is prescribed. Expressions for $S_{x}$ and $S_{y}$ are obtained in the form of a series expansion, grouped into terms depending on their order with respect to $1 / n$, and on the magnitude of applied deformation. Expressions including terms up to the fourth power of $1 / n$ ( $n=$ number of chain repeat units) and eighth power of the extension ratios are derived. Configurational averages appearing in the coefficients of the expression for the orientation functions are obtained by Monte Carlo simulation for a poly(ethylene)-like model chain with 100 bonds. Different levels of approximation for $S_{x}$ and $S_{y}$, comprising terms linear and second order in $1 / n$, are compared. The limits of validity of the expressions are discussed in relation to finite chain extensibility. Results of calculations show that the segmental orientation $S_{x}$ is strongly affected by finite extensions imposed along the transverse direction, $y$. When the strains are made infinitesimally small, however, $S_{x}$ is demonstrated to be uncoupled from the strain applied along the $y$-direction.
\end{abstract}

\section{Introduction}

Molecular aspects of segmental orientation in uniaxially deformed networks have been studied extensively, both theoretically ${ }^{1-4}$ and experimentally. ${ }^{5-17}$ The problem has been investigated at different levels of approximation. Among these, the well-known Kuhn expression for a freely jointed chain model, ${ }^{18}$

$$
S=\frac{1}{5 N}\left(\lambda^{2}-1 / \lambda\right)
$$

has been successful in interpreting experimental data for segmental orientation in uniaxial deformation. Equation 1 relates the second-order Legendre polynomial, or the orientation function, $S$, of the Kuhn segments of a chain to the number $N$ of Kuhn segments and the extension ratio, $\lambda$, in uniaxial deformation. This simple expression, which is the first term of a series expansion ${ }^{19}$ in terms of polynomials of $\lambda$, has later been generalized to the analysis of real chains by Nagai. ${ }^{1}$ Effects of higher order terms resulting from large deformations and short, non-Gaussian chains have been given. ${ }^{4,20}$ On the experimental side, various techniques have been used successfully. Among these are infrared dichroism, ${ }^{6,13-15}{ }^{1} \mathrm{H}-\mathrm{NMR}$ spectroscopy, ${ }^{9-12}$ fluorescence polarization, ${ }^{21,22}$ and wide angle X-ray scattering. ${ }^{16,17}$

The study of segmental orientation in biaxially oriented polymers, on the other hand, both on experimental and theoretical grounds, has been mostly confined to un-cross-linked systems. ${ }^{21,23,24-26}$ This is notably due to the recent technological success of high-performance biaxially oriented polymer films. A detailed molecular theory of biaxial orientation for networks is missing at the present. The aim of the present study is to extend the Nagai formulation of segmental orientation in uniaxially deformed networks to the analysis of biaxially oriented amorphous networks. In this sense the present paper is a continuation of the previous study, ${ }^{4}$ which will be referred to as paper 1 .

\footnotetext{
${ }^{\otimes}$ Abstract published in Advance ACS Abstracts, December 1,
} 1994

0024-9297/95/2228-0582\$09.00/0

\section{Theory}

Model and Assumptions. The molecular model of the present analysis of biaxial orientation and the assumptions are similar to those adopted for uniaxial segmental orientation: (i) The network is affine. The end-to-end vector of each network chain transforms affinely with macroscopic strain. Implicit in this assumption is that the junctions at the ends of the chains do not fluctuate in space. (ii) The network is incompressible. (iii) The segments of the chain do not interact with the surrounding chains in the deformed network and therefore exhibit a phantom-like behavior. Implicit in this assumption is that the segments along the chain contour fluctuate in space, subject only to the constraints imposed by the fixed ends of the chains. The orientation of the segments is a direct consequence of the displacement of the two ends of the chain and is therefore of purely intramolecular origin. (iv) The network is subject to a state of principal homogeneous macroscopic deformation. (v) The distribution of the orientations of chain vectors and segments in the undistorted network is isotropic.

State of Macroscopic and Microscopic Deformation. Biaxial orientation is discussed in terms of the macroscopic deformation tensor, $\lambda$, which is defined as

$$
\lambda=\left[\begin{array}{lll}
\lambda_{x} & 0 & 0 \\
0 & \lambda_{y} & 0 \\
0 & 0 & \frac{1}{\lambda_{x} \lambda_{y}}
\end{array}\right]
$$

Here, $\lambda_{x}$ and $\lambda_{y}$ are the ratios of the final to the undeformed lengths of the sample along the laboratory-fixed $x$ - and $y$-axes. The sample is deformed in the $x y$-plane, $\lambda_{x}$ and $\lambda_{y}$ are the independent variables characterizing the macroscopic deformation. The deformation along the $z$-direction follows from the incompressibility assumption.

As a result of the affine network assumption, the ratios of the mean square averages of the $x_{-}, y_{-}$, and $z$-components of the end-to-end vectors in the deformed state to those in the undeformed state are related to the components of the macroscopic deformation tensor as

$$
\lambda_{x}^{2}=\frac{\left\langle x^{2}\right\rangle}{\left\langle x^{2}\right\rangle_{0}} \quad \lambda_{y}{ }^{2}=\frac{\left\langle y^{2}\right\rangle}{\left\langle y^{2}\right\rangle_{0}} \quad \lambda_{z}^{2}=\frac{\left\langle z^{2}\right\rangle}{\left\langle z^{2}\right\rangle_{0}}
$$

The brackets indicate the ensemble average over all chain 
(a)
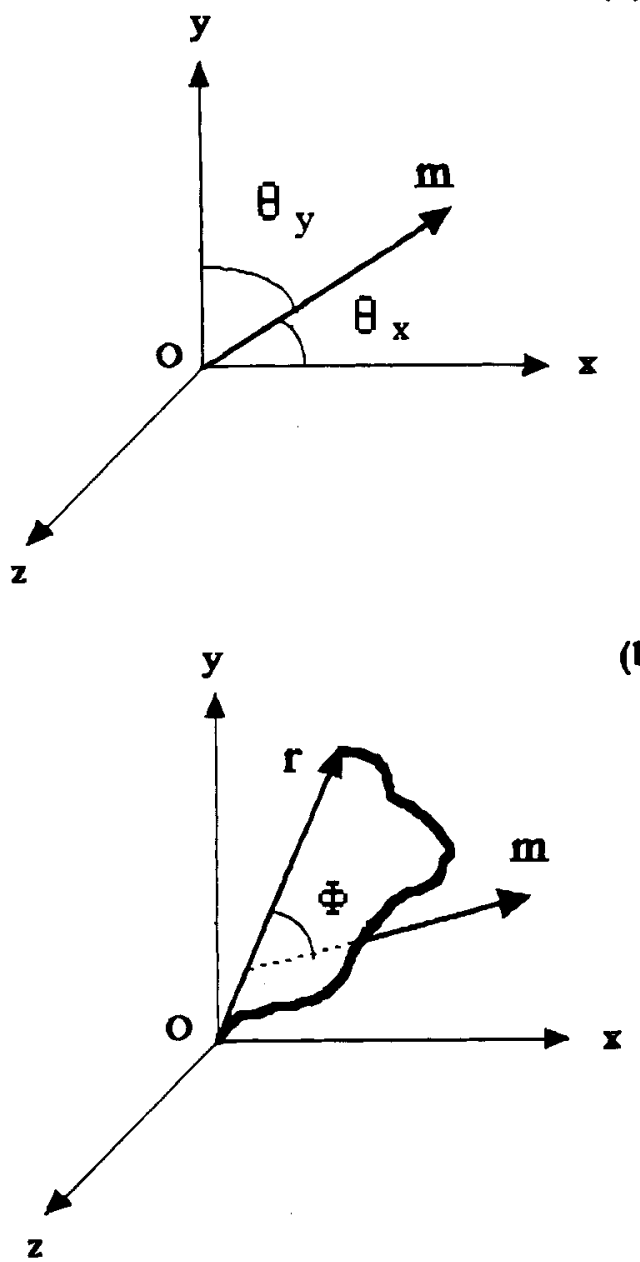

(b)

Figure 1. (a) Schematic representation of the investigated vector $\mathbf{m}$, making angle $\theta_{x}$ with the $x$-axis of the laboratoryfixed frame Oxyz and angle $\theta_{y}$ with the $y$-axis. (b) Schematic representation of the end-to-end vector $\mathbf{r}$ and the investigated vector $\mathbf{m}$, making angle $\Phi$ with $\mathbf{r}$.

configurations, and the subscript 0 represents the undeformed state.

Description of Segmental Orientation. The orientation of a vector, $\mathbf{m}$, affixed to a chain in a network which is biaxially deformed along $x$ - and $y$-directions is investigated. In infrared spectroscopy, $\mathbf{m}$ is usually identified with the transition moment vector whose orientation is being detected experimentally. The orientation of $\mathbf{m}$ with respect to the laboratoryfixed frame Oxyz is described by the angles $\theta_{x}$ and $\theta_{y}$. m makes an angle, $\Phi$, with the instantaneous position of the endto-end vector $\mathbf{r}$, of the chain to which it is rigidly affixed, as shown in Figure 1. Two independent second Legendre functions are used to describe the orientation of $\mathbf{m}$ with respect to the $x$ - and $y$-directions, respectively, as

$$
\begin{gathered}
S_{x}=1 / 2\left[3\left\langle\cos ^{2} \theta_{x}\right\rangle-1\right] \\
S_{y}=1 / 2\left[3\left\langle\cos ^{2} \theta_{y}\right\rangle-1\right]
\end{gathered}
$$

Here, the angular brackets refer to the ensemble average of all chain configurations. Positive $S_{x}$ (or $S_{y}$ ) values correspond to a preferential orientation along the $x$ - (or $y$-) direction, whereas negative $S_{x}$ (or $S_{y}$ ) values indicate a preference for confinement in the plane perpendicular to $x$ - (or $y$-) axes.

Expression of Segmental Orientation Based on the Nagai Formulation. 1,4 The expression for the orientation of a given segment relative to a laboratory-fixed axis may be obtained in its most general form following the formulation by Nagai. The averages $\left\langle\cos ^{2} \theta_{x}\right\rangle$ and $\left\langle\cos ^{2} \theta_{y}\right\rangle$ of eqs 4 and 5 are found in two steps: First, the average orientation of $\mathbf{m}$ relative to $x$ - and $y$-axes for a chain with a fixed end-to-end vector is obtained as $\left(\overline{\cos ^{2} \theta_{x}}\right)_{r}$ and $\left(\overline{\cos ^{2} \theta_{y}}\right)_{r}$. During this averaging, the chain is assumed to take all possible configurations subject only to the constancy of the chain contour length and the end-to-end vector, $\mathbf{r}$. That the chain may take all possible configurations is a consequence of the phantom-like chain assumption. It is to be noted that the use of this assumption limits the problem to systems where intermolecular interactions are small or nonexistent. The present formulation cannot therefore be applied to the analysis of orientation in un-cross-linked bulk polymers below the glass transition temperature where the dominant forces orienting the segments are intermolecular. The second stage of the averaging is performed over all magnitudes and orientations of the endto-end vectors. During this averaging the affine network assumption is employed as a result of which the problem is simplified significantly. The details of the two steps of averaging are as follows.

Step 1: Averaging over all configurations of a chain with fixed $\mathbf{r}$. At this step, the average $\left(\overline{\cos ^{2} \theta_{k}}\right)_{\mathbf{r}}(k=x$ or $y)$ is evaluated. The expression for this average in real chains follows from paper 1 as

$$
\begin{array}{r}
\left(\cos ^{2} \theta_{k}\right)_{\mathbf{r}}=1 / 3+\left(1-\beta_{1 k} g_{4}-\beta_{2 k} g_{6}-\beta_{3 k} g_{8}+\ldots\right) \times \\
\sum_{i}\left(\alpha_{i k} \eta_{2 i} / 3\right)
\end{array}
$$

for $k=x$ or $y$. There are two groups of variables of different character on the right-hand side of eq 6 . (i) $\eta_{i}$ and $g_{i}$. These depend on the average configurational properties of the network chains in the undeformed state and are independent of the state of deformation. They are expressed in terms of the moments $\left\langle r^{2 m}\right\rangle_{0}(1 \leq m \leq 4)$ and $\left\langle r^{2 m} \cos ^{2} \Phi\right\rangle_{0}$ of the endto-end vector $\mathbf{r}$. Expressions for these variables have been given in paper 1 and are also reproduced in Appendix 1 for completeness. The parameters $g_{i}$ and $\eta_{i}$ scale with the number of segments, $n$, in the chain $a^{2,4}$

$$
\begin{gathered}
g_{4}, \eta_{2} \sim n^{-1} \\
g_{6}, g_{8}, \eta_{4}, \eta_{6} \sim n^{-2} \\
\eta_{8} \sim n^{-3}
\end{gathered}
$$

(ii) $\alpha_{i}$ and $\beta_{i}$. These variables depend on the particular chain conformation and therefore assume different values depending on the state of macroscopic deformation. Explicit expressions for $\alpha_{i}$ and $\beta_{i}$ are presented in paper 1 and Appendix 2. The full expression given by eq 6 is truncated at different terms, depending on the order of approximation used in the treatment.

Step 2: Averaging over all chains in the deformed network At this step, the expression given by eq 6 has to be averaged over the ensemble of chains with various orientations and magnitudes of end-to-end vectors. The resulting average is denoted as $\left\langle\cos ^{2} \theta_{k}\right\rangle$, where $k=x$ or $y$. This step requires the evaluation of the terms $\left\langle\alpha_{i k}\right\rangle$ and $\left\langle\alpha_{i k} \beta_{j k}\right\rangle$, conforming with eq 6. The explicit forms of these averages were derived in paper 1 for the case of uniaxial deformation. For biaxial orientation, by following the procedure adopted in paper 1, the ensemble averages $\left\langle\alpha_{i k}\right\rangle$ are obtained for $1 \leq i \leq 4$ from eqs $2-1$ and $2-2$ in Appendix 2 and their products as

$$
\begin{gathered}
\left\langle\alpha_{1 k}\right\rangle=2 \Lambda_{2 k} \\
\left\langle\alpha_{2 k}\right\rangle=14 \Lambda_{2 k}-\frac{18}{5} \frac{\left\langle r^{4}\right\rangle_{0}}{\left\langle r^{2}\right\rangle_{0}^{2} \Lambda_{4 k}} \\
\left\langle\alpha_{3 k}\right\rangle=126 \Lambda_{2 k}-\frac{324}{5} \frac{\left\langle r^{4}\right\rangle_{0}}{\left\langle r^{2}\right\rangle_{0}^{2}} \Lambda_{4 k}+\frac{54}{7} \frac{\left\langle r^{6}\right\rangle_{0}}{\left\langle r^{2}\right\rangle_{0}^{3}} \Lambda_{6 k}
\end{gathered}
$$




$$
\begin{aligned}
\left\langle\alpha_{4 k}\right\rangle=1386 \Lambda_{2 k}-\frac{5346}{5} \frac{\left\langle r^{4}\right\rangle_{0}}{\left\langle r^{2}\right\rangle_{0}^{2}} \Lambda_{4 k}+ & \\
& \frac{1782}{7} \frac{\left\langle r^{6}\right\rangle_{0}}{\left\langle r^{2}\right\rangle_{0}^{3}} \Lambda_{6 k}-18 \frac{\left\langle r^{8}\right\rangle_{0}}{\left\langle r^{2}\right\rangle_{0}^{4}} \Lambda_{8 k}
\end{aligned}
$$

Likewise, the ensemble averages $\left\langle\alpha_{i k} \beta_{j k}\right\rangle$ appearing in the expansion for $\left\langle\cos ^{2} \theta_{k}\right\rangle$, after truncating terms scaling with (1/ $n)^{a}$ where $a>3$, are expressed as

$$
\begin{aligned}
& \left\langle\alpha_{1 k} \beta_{1 k}\right\rangle=30 \Lambda_{2 k}-36 \frac{\left\langle r^{4}\right\rangle_{0}}{\left\langle r^{2}\right\rangle_{0}{ }^{2}} \Lambda_{4 k}+\frac{54}{7} \frac{\left\langle r^{6}\right\rangle_{0}}{\left\langle r^{2}\right\rangle_{0}{ }^{3}} \Lambda_{6 k} \\
& \left\langle\alpha_{1 k} \beta_{2 k}\right\rangle=210 \Lambda_{2 k}-378 \frac{\left\langle r^{4}\right\rangle_{0}}{\left\langle r^{2}\right\rangle_{0}{ }^{2}} \Lambda_{4 k}+ \\
& 162 \frac{\left\langle r^{6}\right\rangle_{0}}{\left\langle r^{2}\right\rangle_{0}^{3}} \Lambda_{6 k}-18 \frac{\left\langle r^{8}\right\rangle_{0}}{\left\langle r^{2}\right\rangle_{0}^{4}} \Lambda_{8 k} \\
& \left\langle\alpha_{1 k} \beta_{3 k}\right\rangle=1890 \Lambda_{2 k}-4536 \frac{\left\langle r^{4}\right\rangle_{0}}{\left\langle r^{2}\right\rangle_{0}{ }^{2}} \Lambda_{4 k}+ \\
& 2916 \frac{\left\langle r^{6}\right\rangle_{0}}{\left\langle r^{2}\right\rangle_{0}{ }^{3}} \Lambda_{6 k}-648 \frac{\left\langle r^{8}\right\rangle_{0}}{\left\langle r^{2}\right\rangle_{0}{ }^{4}} \Lambda_{8 k} \\
& \left\langle\alpha_{2 k} \beta_{1 k}\right\rangle=210 \Lambda_{2 k}-306 \frac{\left\langle r^{4}\right\rangle_{0}}{\left\langle r^{2}\right\rangle_{0}^{2}} \Lambda_{4 k}+ \\
& \frac{918}{7} \frac{\left\langle r^{6}\right\rangle_{0}}{\left\langle r^{2}\right\rangle_{0}{ }^{3}} \Lambda_{6 k}-18 \frac{\left\langle r^{8}\right\rangle_{0}}{\left\langle r^{2}\right\rangle_{0}{ }^{4}} \Lambda_{8 k} \\
& \left\langle\alpha_{3 k} \beta_{1 k}\right\rangle=1890 \Lambda_{2 k}-3240 \frac{\left\langle r^{4}\right\rangle_{0}}{\left\langle r^{2}\right\rangle_{0}^{2}} \Lambda_{4 k}+ \\
& \frac{13932}{7} \frac{\left\langle r^{6}\right\rangle_{0}}{\left\langle r^{2}\right\rangle_{0}{ }^{3}} \Lambda_{6 k}-504 \frac{\left\langle r^{8}\right\rangle_{0}}{\left\langle r^{2}\right\rangle_{0}{ }^{4}} \Lambda_{8 k}
\end{aligned}
$$

$\Lambda_{2 k}, \Lambda_{4 k}, \Lambda_{6 k}$, and $\Lambda_{8 k}$ in eqs 8 and 9 are polynomials in the components of the macroscopic deformation tensor. Explicit expressions for these polynomials, referred to as deformation functions hereafter, are

$$
\begin{aligned}
& \Lambda_{2 x}=\lambda_{x}{ }^{2}-1 / 2\left(\lambda_{y}{ }^{2}\right)-1 / 2\left(\lambda_{x} \lambda_{y}\right)^{-2} \\
& \Lambda_{4 x}=\lambda_{x}{ }^{4}-1 / 2\left(\lambda_{y}{ }^{4}\right)+1 / 6\left(\lambda_{x} \lambda_{y}\right)^{2}-1 / 3 \lambda_{x}{ }^{-2}+1 / 6 \lambda_{y}{ }^{-2}- \\
& 1 / 2\left(\lambda_{x} \lambda_{y}\right)^{-4} \\
& \Lambda_{6 x}=\lambda_{x}{ }^{6}-1 / 2\left(\lambda_{y}{ }^{6}\right)-1 / 2\left(\lambda_{x} \lambda_{y}\right)^{-6}+{ }^{3} / 10\left(\lambda_{x}{ }^{4} \lambda_{y}{ }^{2}\right)+ \\
& { }^{3 / 10}\left(\lambda_{x}{ }^{2} \lambda_{y}{ }^{-2}\right)-3 / 10\left(\lambda_{x}{ }^{-2} \lambda_{y}{ }^{2}\right)-{ }^{3} / 10\left(\lambda_{x}{ }^{-4} \lambda_{y}{ }^{-2}\right) \\
& \Lambda_{8 x}=\lambda_{x}^{8}-1 / 2\left(\lambda_{y}{ }^{8}\right)-1 / 2\left(\lambda_{x} \lambda_{y}\right)^{-8}+{ }^{5} / 14\left(\lambda_{x}{ }^{6} \lambda_{y}{ }^{2}\right)+ \\
& { }^{9} /{ }_{70}\left(\lambda_{x}{ }^{4} \lambda_{y}{ }^{4}\right)-1 / 14\left(\lambda_{x}{ }^{2} \lambda_{y}{ }^{6}\right)+{ }^{5} /{ }_{14}\left(\lambda_{x}{ }^{4} \lambda_{y}{ }^{-2}\right)+{ }^{3} /{ }_{35} \lambda_{x}{ }^{2}-{ }^{3} /{ }_{70} \lambda_{y}{ }^{2}- \\
& 2 / 7\left(\lambda_{x}{ }^{-2} \lambda_{y}{ }^{4}\right)+9 / 70_{y} \lambda^{-4}-9 /{ }_{35} \lambda_{x}{ }^{-4}-3 / 70\left(\lambda_{x}{ }^{-2} \lambda_{y}{ }^{-2}\right)- \\
& 1 / 14\left(\lambda_{x}{ }^{-4} \lambda_{y}{ }^{-6}\right)-{ }^{2} /{ }_{7}\left(\lambda_{x}{ }^{-6} \lambda_{y}{ }^{-4}\right)
\end{aligned}
$$

for the case $k=x$. Expressions for $\Lambda_{i y}(i=2,4,6$, and 8$)$ are readily obtained by exchanging the subscripts $x$ and $y$ in eq 10.

Substituting the ensemble averages given by eq 9 , as well as eqs 1-1 and 1-2 of Appendix 1, into eq 6 leads to the average square cosine of the angle between the vectors $\mathbf{m}$ and the laboratory-fixed axes, $x$ or $y$. The resulting expression is organized as

$$
\begin{array}{r}
\left.\left\langle\cos ^{2} \theta_{k}\right\rangle \equiv\left\langle\overline{\left(\cos ^{2} \theta_{k}\right.}\right)_{\mathbf{r}}\right\rangle=1 / 3\left[1+D_{1} \Lambda_{2 k}+D_{2} \Lambda_{4 k}+D_{3} \Lambda_{6 k}+\right. \\
\left.D_{4} \Lambda_{8 k}+\ldots\right](11)
\end{array}
$$

where the coefficients $D_{i}$, referred to as the $i$ th configurational factor for segmental orientation, are given for $1 \leq i \leq 4$ by

$$
\begin{aligned}
& D_{1}=2 \eta_{2}+14 \eta_{4}+126 \eta_{6}+1386 \eta_{8}-30 \eta_{2} g_{4}-210 \eta_{2} g_{6}- \\
& 1890 \eta_{2} g_{8}-210 \eta_{4} g_{4}-1890 \eta_{6} g_{4} \\
& D_{2}=-\frac{18}{5}\left(\eta_{4}+18 \eta_{6}+297 \eta_{8}-10 \eta_{2} g_{4}-105 \eta_{2} g_{6}-\right. \\
& \left.1260 \eta_{2 g_{8}}-85 \eta_{4} g_{4}-900 \eta_{6} g_{4}\right) \frac{\left\langle r^{4}\right\rangle_{0}}{\left\langle r^{2}\right\rangle_{0}^{2}} \\
& D_{3}=\frac{54}{7}\left(\eta_{6}+33 \eta_{8}-\eta_{2} g_{4}-21 \eta_{2} g_{6}-378 \eta_{2 g_{8}}-17 \eta_{4} g_{4}-\right. \\
& 258 \eta_{6} g_{4} \frac{\left\langle r^{6}\right\rangle_{0}}{\left\langle r^{2}\right\rangle_{0}^{3}} \\
& D_{4}=18\left(\eta_{2 g_{6}}+36 \eta_{2 g_{8}}+\eta_{4} g_{4}+28 \eta_{6} g_{4} \frac{\left\langle r^{8}\right\rangle_{0}}{\left\langle r^{2}\right\rangle_{0}{ }^{4}}\right.
\end{aligned}
$$

The coefficients $D_{i}(1 \leq i \leq 4)$ are functions of only chain constitution and otherwise do not depend on the state of deformation. ${ }^{26}$ In eq 11 , the terms are grouped so as to give the leading term of $D_{1}$ of order $n^{-1}$, the leading terms of $D_{2}$ and $D_{3}$ of order $n^{-2}$, and that of $D_{4}$ of order $n^{-3}$. Replacing eq 11 into eqs 4 and 5 leads to the orientation function, $S_{k}(k=$ $x$ or $y$ ), in terms of $D_{i}$ and $\Lambda_{2 i k}$ as

$$
S_{k}=1_{2}\left[D_{1} \Lambda_{2 k}+D_{2} \Lambda_{4 k}+D_{3} \Lambda_{6 k}+D_{4} \Lambda_{8 k}+\ldots\right]
$$

Different Levels of Approximation. The state of segmental orientation at various levels of approximation may be obtained from eq 13 by retaining the relevant terms in the brackets. These terms consist of products of two factors, the configurational factors $D_{i}$ and the deformation functions $\Lambda_{2 i k}$. The former are functions of average chain dimensions in the unperturbed state. Terms with larger subscript $i$ become smaller with increasing chain length. $\Lambda_{2 i k}$ 's are functions of the macroscopic state of deformation. Those with larger subscript $i$ are of higher order in extension ratios and therefore become much smaller at small deformations. There is no clearcut criterion for estimating how many of the terms in eq 13 should be retained in different situations. In neglecting the higher order $D_{i}$ 's, one may adopt the plausible criterion that the chains should be sufficiently long to behave as Gaussian. For poly(ethylene) chains, for example, Gaussian behavior is obtained when the number of repeat units exceeds ca. 50 or $60.27,28$ For neglecting higher order $\Lambda_{2 i k}$ 's, on the other hand, the deformation should be sufficiently small. In this case, one should consider the degree of stretch of the chain under a given macroscopic deformation with the finite extensibility of the chain. In uniaxial extension, finite extensibility constraints operate when the macroscopic extension ratio reaches the maximum value, $\lambda_{\max , u}$. The latter is related to chain parameters by

$$
\lambda_{\max , u}=\frac{r_{\max }}{\left\langle r^{2}\right\rangle_{0}{ }^{1 / 2}}=\frac{n l \cos \Theta / 2}{\left\langle r^{2}\right\rangle_{0}^{1 / 2}}
$$

Here, $r_{\max }$ is the maximum elongation of the chain with alltrans configuration, $\left\langle r^{2}\right\rangle_{0}^{1 / 2}$ is the mean square end-to-end vector of unperturbed chains, $l$ is the bond length, and $\Theta$ is the supplemental bond angle. For the freely rotating bond model chain of 100 bonds, which will be used in the present simulations for example, eq 14 gives $\lambda_{\max }=5.83$. A relationship of maximum extensibility of chains to the macroscopic state of biaxial deformation cannot be uniquely obtained, however, due to the presence of two independent variables, $\lambda_{x}$ and $\lambda_{y}$. For the biaxial case, one may adopt the plausible assumption that the first strain invariant is the same under 
Table 1. Moments Calculated from Monte Carlo Simulation

\begin{tabular}{ccc}
\hline$m$ & $\left\langle r^{2 m}\right\rangle_{0}\left(\AA^{2}\right)$ & $\left\langle r^{2 m} \cos ^{2} \Phi\right\rangle_{0}\left(\AA^{2}\right)$ \\
\hline 1 & $4.684 \times 10^{2}$ & $1.600 \times 10^{2}$ \\
2 & $3.592 \times 10^{5}$ & $1.239 \times 10^{5}$ \\
3 & $3.779 \times 10^{8}$ & $1.316 \times 10^{8}$ \\
4 & $5.004 \times 10^{11}$ & $1.758 \times 10^{11}$
\end{tabular}

uniaxial and biaxial deformation when the maximum extensibility of the chains is reached. Thus,

$$
\begin{aligned}
I_{1, \max } & \equiv \lambda_{x}{ }^{2}+\lambda_{y}{ }^{2}+\lambda_{z}{ }^{2} \\
& =\lambda_{\max , u}^{2}+\frac{2}{\lambda_{\max , u}}=\lambda_{\max , x}^{2}+\lambda_{\max , y}^{2}+\frac{1}{\lambda_{\max , x}^{2} \lambda_{\max , y}^{2}}
\end{aligned}
$$

where $I_{1, \max }$ is the first strain invariant when the chains reach their finite extensibility limits, $\lambda_{\max , u}$ is given by eq 14 , and $\lambda_{\max x}$ and $\lambda_{\max , y}$ are the maximum extension ratios in biaxial deformation. Since the maximum extension ratios will be considerably larger than unity, eq $\mathbf{1 5}$ may be approximated by

$$
\lambda_{\max , x}^{2}+\lambda_{\max , y}^{2} \approx \frac{n^{2} l^{2} \cos ^{2} \Theta / 2}{\left\langle r^{2}\right\rangle_{0}}
$$

We note that for the freely rotating model chain of 100 bonds, the right-hand side of eq 16 equates to 34 .

For sufficiently long chains under relatively small deformations, one may use the first-order approximation by choosing the following set of coefficients:

$$
\begin{gathered}
D_{1}=2 \eta_{2} \\
D_{2}=D_{3}=D_{4}=0
\end{gathered}
$$

For higher extension ratios or shorter chains, the secondorder approximation is applied by choosing the following set of coefficients:

$$
\begin{gathered}
D_{1}=2 \eta_{2}+14 \eta_{4}+126 \eta_{6}-30 \eta_{2 g_{4}} \\
D_{2}=-\frac{18}{5}\left(\eta_{4}+18 \eta_{6}-10 \eta_{25} g_{4}\right) \frac{\left\langle r^{4}\right\rangle_{0}}{\left\langle r^{2}\right\rangle_{0}^{2}} \\
D_{3}=\frac{54}{7}\left(\eta_{6}-\eta_{25} g_{4}\right) \frac{\left\langle r^{6}\right\rangle_{0}}{\left\langle r^{2}\right\rangle_{0}^{3}} \\
D_{4}=0
\end{gathered}
$$

Here, $D_{1}$ scales as $n^{-1}$ and $D_{2}$ and $D_{3}$ scale as $n^{-2}$

The infinitesimally small deformation limit may be obtained by substituting $\epsilon_{x}=\lambda_{x}-1$ and $\epsilon_{y}=\lambda_{y}-1$ into eq 10 , retaining the linear terms only in the numerators by taking $\left(1+\epsilon_{x}\right)^{m} \approx$ $1+m \epsilon_{x}$, and inserting the resulting deformation functions into eq 13. The resulting expression for segmental orientation turns out to be identical in form to that obtained in paper 1 for uniaxial deformation, i.e.,

$$
S_{k}=3 / 2\left(D_{1}+7 / 3 D_{2}+21 / 5 D_{3}+33 / 5 D_{4}+\ldots\right) \epsilon_{k}
$$

for $k=x$ or $y$. This unexpected result indicates that segmental orientation $S_{k}$ along the $k$-direction for infinitesimal biaxial deformations is a function of the strain $\epsilon_{k}$ along this direction only and is uncoupled to the imposed strain along the other direction.

Calculations. The dependence of biaxial orientation on the state of macroscopic deformation and chain parameters is investigated in this section for a model chain of 100 bonds. The configurational averages appearing in the coefficients $D_{i}$ are calculated by the Monte Carlo technique as described before ${ }^{4,20}$ The choice of the Monte Carlo technique rather than the exact generator matrix multiplication scheme ${ }^{25,29}$ is dictated by the presence of the higher order moments in $D_{i}$.
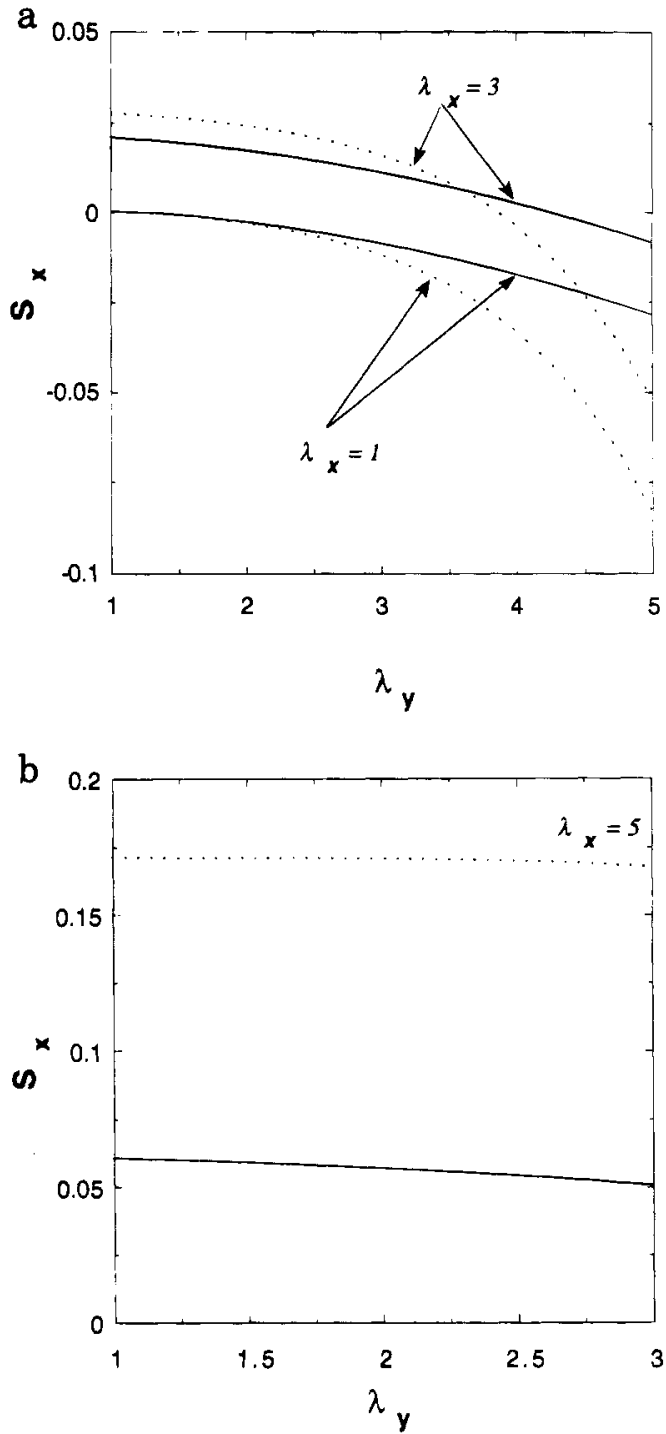

Figure 2. (a) Dependence of $S_{x}$ on the extension ratio along the $y$-direction, $\lambda_{y}$, for given $\lambda_{x}$ 's. The solid curves represent the first-order approximation, evaluated with eqs 13 and 17. The dotted curves are obtained with the second-order approximation, by inserting eqs 18 into eq 13. (b) Dependence of $S_{x}$ on $\lambda_{y}$ for $\lambda_{x}=5$. See legend of panel a.

Following conventional chain generation methods, bond-based coordinate systems are defined for skeletal bonds and the 0th atom is placed at the origin. The length of the $\mathrm{C}-\mathrm{C}$ bonds is taken as $1.53 \AA$, and the supplemental bond angle $\angle \mathrm{CCC}$ is $70.53^{\circ}$. Three rotational isomeric states, trans $(\mathrm{t})$, gauche $\left(\mathrm{g}^{+}\right)$, and gauche $\left(\mathrm{g}^{-}\right)$with respective torsional angles of $0^{\circ}, 120^{\circ}$, and $-120^{\circ}$, are assigned with equal probability to backbone bonds, conforming with the freely rotating model of chain statistics. The moments $\left\langle\mathrm{r}^{2 \mathrm{~m}}\right\rangle_{0}$ and $\left\langle\mathrm{r}^{2 \mathrm{~m}} \cos ^{2} \boldsymbol{\Phi}\right\rangle_{0}$ are evaluated over an ensemble of $10^{5}$ Monte Carlo chains; the resulting values for $m=1-4$ are listed in Table 1 .

\section{Results and Discussion}

The orientation functions $S_{x}$ and $S_{y}$ have been investigated as functions of the biaxial state of deformation in terms of the first- and second-order approximations, using eqs 17 and 18 , respectively.

In Figure 2, the dependence of $S_{x}$ on the extension ratio $\lambda_{y}$ along the lateral direction is presented for three different values of extension ratios $\lambda_{x}$ along the reference direction. $\lambda_{x}$ is taken as 1 and 3 in Figure $2 a$ and 5 in Figure 2b. In these figures, the solid lines represent the first-order approximation and the dashed lines the second-order approximation. The maximum value of 

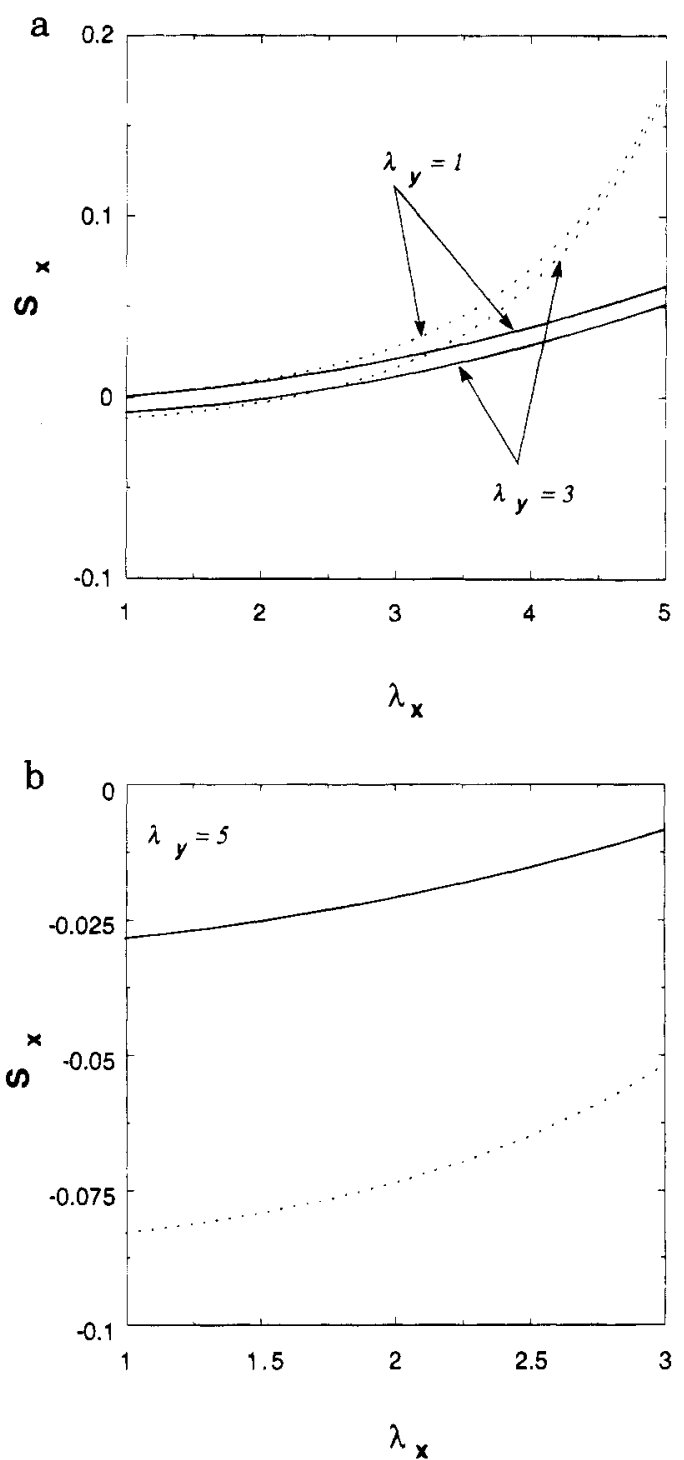

Figure 3. (a) Dependence of $S_{x}$ on $\lambda_{x}$ for $\lambda_{y}=1$ and 3. See legend of Figure 2a. (b) Dependence of $S_{x}$ on $\lambda_{x}$ for $\lambda_{y}=5$. See legend of Figure 2a.

the abscissa, $\lambda_{y}=5$, in Figure $2 \mathrm{a}$ is chosen according to eq 16 so that maximum extensibility of the chains is not exceeded when $\lambda_{x}=3$. Similarly, in Figure $2 b$, the abscissa range is chosen as $\lambda_{y} \leq 3$ so that the maximum extensibility is not exceeded when $\lambda_{x}=5$. As expected, larger $\lambda_{x}$ values lead to higher orientation along the $x$-direction, whereas with decreasing $\lambda_{x}$ and increasing $\lambda_{y}$ a preferential alignment along the lateral direction is indicated by the negative $S_{x}$ values. In Figure 2a, the first- and second-order approximations are close to each other in the range $\lambda_{y}<3$. For both approximations, $S_{x}$ decreases with $\lambda_{y}$, as expected. The decrease in the second-order approximation is very strong in the range $3<\lambda_{y}<5$, which indicates the inadequacy of the first-order approximation for treating segmental orientation as the deformation is increased. This feature becomes even more pronounced in Figure 2b, in which the dependence of $S_{x}$ on $\lambda_{y}$ is shown for $\lambda_{x}=5$. The difference between the results of the first and second approximations is significant. In fact, the extent of orientation along the $x$-direction is seriously underestimated by adopting the first-order approximation.

In Figure 3, $S_{x}$ is plotted against $\lambda_{x}$ for three different values of $\lambda_{y}$. In Figure 3a, the curves obtained with the first- and second-order approximations are almost in- distinguishable in the range $\lambda_{x}<3$. The orientation functions increase slowly in this region and exhibit a weak dependence on $\lambda_{y}$. In the range $3<\lambda_{x}<5$, however, the second-order approximation shows an abrupt increase while the first-order approximation curves continue to increase smoothly. An interesting feature observed in Figure 3a is that at high values of $\lambda_{x}, S_{x}$ values for $\lambda_{y}=1$ and 3 approach each other. In Figure $3 b, S_{x}$ is represented as a function of $\lambda_{x}$ when $\lambda_{y}$ is kept at a constant value of 5 . A significant difference is observed between the orientation functions predicted by the first- and second-order approximations. It is clear that for such large extension ratios along the lateral direction $\left(\lambda_{y}=5\right)$ there will be a negative orientation with respect to the reference direction $\left(S_{x}\right.$ $<0$ ), i.e., chain segments would tend to be confined in a plane perpendicular to the $x$-axis. Again, the absolute value in the orientation function $S_{x}$ is considerably underestimated upon adoption of the first-order approximation. The second-order approximation gives much lower values than the first-order, but the two curves have nearly the same slope.

In Figure $4, S_{x}$ and $S_{y}$ are plotted for compressive deformation along the $y$-direction with $\lambda_{y}$ changing between 0.2 and 1.0. The solid lines result from the first-order approximation and the dashed lines from the second-order approximation. We note the difference between the two scales for $S_{x}$ and $S_{y}$ on the left and right ordinates, respectively, which indicates that $S_{y}$ is much more sensitive to variations in $\lambda_{y}$, compared to $S_{x}$, at low $\lambda_{x}$ values (Figure 4a), while the opposite behavior takes place at high $\lambda_{x}$ values (FIgure 4c). In Figure 4a, $\lambda_{x}$ is kept contant at 1 . For $\lambda_{y}$ larger than roughly 0.5 , the first- and second-order approximations give identical results for both $S_{x}$ and $S_{y}$. In Figure $4 \mathrm{~b}, S_{x}$ and $S_{y}$ are plotted keeping $\lambda_{x}$ constant at 3 . Throughout the whole $\lambda_{y}$ range, since the network is under compression in the $y$-direction, $\boldsymbol{S}_{x}$ has positive values and $\boldsymbol{S}_{y}$ has negative values. In Figure $4 c, \lambda_{x}$ is kept fixed at 5 and $\lambda_{y}$ is varied in the compression range shown. The first-order approximation gives again lower values for $S_{x}$ with respect to second-order approximation. $S_{x}$ is almost insensitive to $\lambda_{y}$, but $S_{y}$ shows a slight increase with increasing $\lambda_{y}$. Comparison of panels a-c in Figure 4 shows that as $\lambda_{x}$ increases from 1 to 3 the orientation increases along the $x$-direction and decreases along the $y$-direction. At a given $\lambda_{x}, S_{x}$ remains almost constant or changes slightly for $0.5<\lambda_{y}<1.0$. As $\lambda_{x}$ increases, the difference between the first- and second-order approximations increases. So the importance of the higher order approximations for high extensions can be again emphasized.

\section{Conclusion}

The Nagai formulation of segmental orientation in amorphous polymer networks is extended to the biaxial state of deformation, and the results of first- and secondorder approximations are investigated in some detail. Examination of Figures 2 and 3 in this respect shows that the first-order approximation may be adopted for biaxial state of strain with sufficient accuracy for $\lambda_{x}<$ 2 and $\lambda_{y}<2$. The limits of macroscopically applied extension ratios are discussed in relation to finite chain extensibility. The formulation, which is in general valid for finite extensions, is simplified for the case of infinitesimal deformations, leading to the interesting result that segmental orientation with respect to a given direction is a function of strain applied in that direction only. 

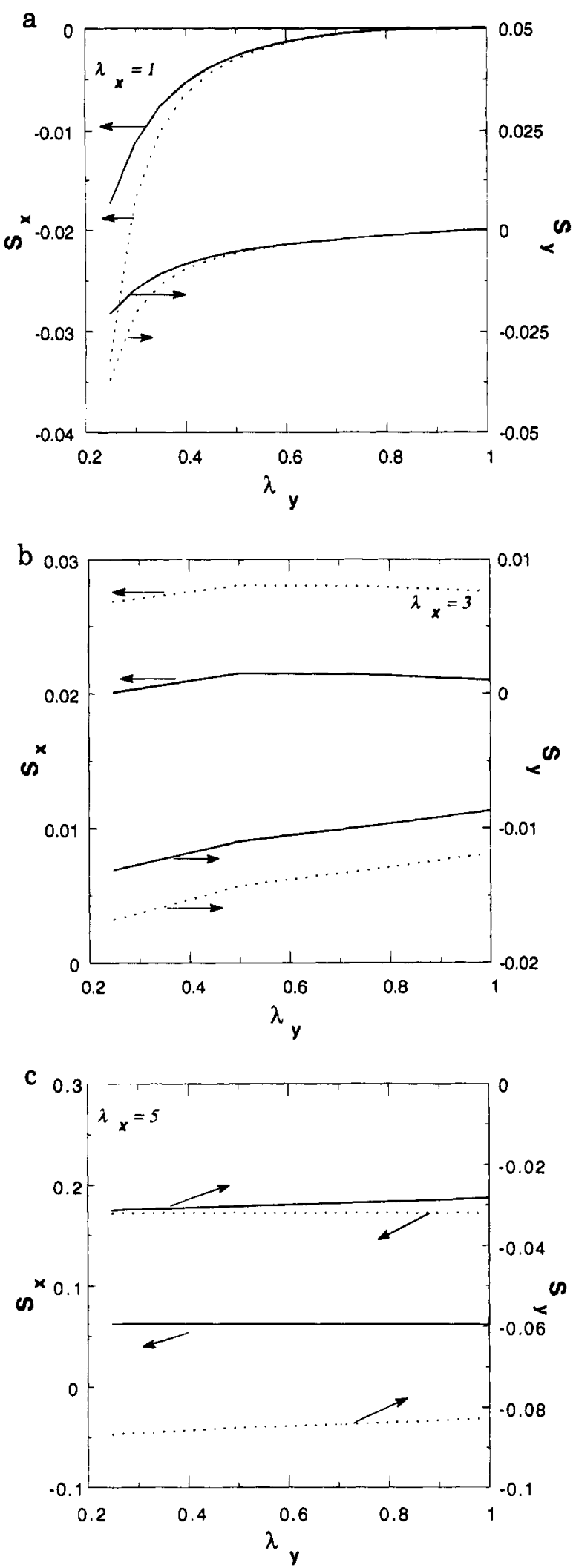

Figure 4. (a) Dependence of $S_{x}$ (left ordinate) and $S_{y}$ (right ordinate) on $\lambda_{y}$ with $\lambda_{x}=1$. Network chains are compressed along the $y$-direction. The solid lines are for first-order approximation, and the dashed lines are for second-order approximation. The arrows indicate the ordinate to which each curve belongs. (b) Dependence of $S_{x}$ and $S_{y}$ on $\lambda_{y}$ with $\lambda_{x}$ $=3$. See legend of panel a. (c) Dependence of $S_{x}$ and $S_{y}$ on $\lambda_{y}$ with $\lambda_{x}=5$. See legend of panel a.

The present theory is based on the phantom-like chain model. As stated in the description of the model, the major consequence of this assumption is that the chain segments take all configurations, subject only to the

constraints imposed by fixing the chain ends. However, both theoretical and experimental work show that intermolecular interactions along the chain contour may contribute to segmental orientation at various levels of importance. $8,30-32$ These effects are not taken into consideration in the present study. It is to be noted that the excess orientation, resulting from entanglements, diminishes strongly upon dilution with a suitable isotropic solvent. Also, increasing chain flexibility and the degree of cross-linking strongly diminishes the contribution of entanglements on segmental orientation.

Acknowledgment. Partial support from Bogazici University Research Funds Grant No. 94P0002 is gratefully acknowledged.

\section{Appendix 1. Configurational Parameters $\eta_{i}$ and $g_{i}$ of the Nagai Theory}

The variables $\eta_{i}$ and $g_{i}$ in eq 6, appearing up to thirdorder approximation, read as follows:

$$
\begin{gathered}
\eta_{2} \equiv \frac{1}{10}\left(\frac{3\left\langle r^{2} \cos ^{2} \Phi\right\rangle_{0}}{\left\langle r^{2}\right\rangle_{0}}-1\right) \\
\eta_{4} \equiv \frac{1}{20}\left[\left(\frac{3\left\langle r^{2} \cos ^{2} \Phi\right\rangle_{0}}{\left\langle r^{2}\right\rangle_{0}}-1\right)-\frac{3}{7}\left(\frac{3\left\langle r^{4} \cos ^{2} \Phi\right\rangle_{0}}{\left\langle r^{2}\right\rangle_{0}^{2}}-\right.\right. \\
\left.\left.\frac{\left\langle r^{4}\right\rangle_{0}}{\left\langle r^{2}\right\rangle_{0}^{2}}\right)\right] \\
\eta_{6} \equiv \frac{1}{80}\left[\left(\frac{3\left\langle r^{2} \cos ^{2} \Phi\right\rangle_{0}}{\left\langle r^{2}\right\rangle_{0}}-1\right)-\frac{6}{7}\left(\frac{3\left\langle r^{4} \cos ^{2} \Phi\right\rangle_{0}}{\left\langle r^{2}\right\rangle_{0}^{2}}-\right.\right. \\
\left.\left.\frac{\left\langle r^{4}\right\rangle_{0}}{\left\langle r^{2}\right\rangle_{0}^{2}}\right)+\frac{1}{7}\left(\frac{3\left\langle r^{6} \cos ^{2} \Phi\right\rangle_{0}}{\left\langle r^{2}\right\rangle_{0}^{3}}-\frac{\left\langle r^{6}\right\rangle_{0}}{\left\langle r^{2}\right\rangle_{0}^{3}}\right)\right] \\
\eta^{8} \equiv \frac{1}{480}\left[\left(\frac{3\left\langle r^{2} \cos ^{2} \Phi\right\rangle_{0}}{\left\langle r^{2}\right\rangle_{0}}-1\right)-\frac{9}{7}\left(\frac{3\left\langle r^{4} \cos ^{2} \Phi\right\rangle_{0}}{\left\langle r^{2}\right\rangle_{0}^{2}}-\right.\right. \\
\left.\frac{\left\langle r^{4}\right\rangle_{0}}{\left\langle r^{2}\right\rangle_{0}^{2}}\right)+\frac{3}{7}\left(\frac{3\left\langle r^{6} \cos ^{2} \Phi\right\rangle_{0}}{\left\langle r^{2}\right\rangle_{0}^{3}}-\frac{\left\langle r^{6}\right\rangle_{0}}{\left\langle r^{2}\right\rangle_{0}^{3}}\right)- \\
\frac{3}{77}\left(\frac{3\left\langle r^{8} \cos ^{2} \Phi\right\rangle_{0}}{\left.\left.\left\langle r^{2}\right\rangle_{0}{ }^{4}-\frac{\left\langle r^{8}\right\rangle_{0}}{\left\langle r^{2}\right\rangle_{0}{ }^{4}}\right)\right]}\right](1-1)
\end{gathered}
$$

and

$$
\begin{gathered}
g_{4} \equiv-\left(\frac{1}{2 ! 2^{2}}\right)\left[1-\frac{3\left\langle r^{4}\right\rangle_{0}}{5\left\langle r^{2}\right\rangle_{0}^{2}}\right] \\
g_{6} \equiv-\left(\frac{1}{3 ! 2^{3}}\right)\left[3\left(1-\frac{3\left\langle r^{4}\right\rangle_{0}}{5\left\langle r^{2}\right\rangle_{0}^{2}}\right)-\left(1-\frac{3^{2}\left\langle r^{6}\right\rangle_{0}}{5.7\left\langle r^{2}\right\rangle_{0}^{3}}\right)\right] \\
g_{8} \equiv-\left(\frac{1}{4 ! 2^{4}}\right)\left[6\left(1-\frac{3\left\langle r^{4}\right\rangle_{0}}{5\left\langle r^{2}\right\rangle_{0}^{2}}\right)-4\left(1-\frac{3^{2}\left\langle r^{6}\right\rangle_{0}}{5.7\left\langle r^{2}\right\rangle_{0}^{3}}\right)+\right. \\
\left.\left(1-\frac{3^{3}\left\langle r^{8}\right\rangle_{0}}{5.7 .9\left\langle r^{2}\right\rangle_{0}^{4}}\right)\right]
\end{gathered}
$$




\section{Appendix 2. r Dependent Parameters $\alpha_{i}$ and $\beta_{i}$ of Eq 6}

The parameters $\alpha_{i}$ and $\beta_{i}$ depend on the specific chain conformation in the deformed state. These are given by

$$
\begin{gathered}
\alpha_{1}=3\left(3 x^{2}-r^{2}\right) /\left\langle r^{2}\right\rangle_{0} \\
\alpha_{2} \equiv 3^{2}\left(\frac{7}{3}-\frac{r^{2}}{\left\langle r^{2}\right\rangle_{0}}\right)\left(3 x^{2}-r^{2}\right) /\left\langle r^{2}\right\rangle_{0} \\
\alpha_{3} \equiv 3^{3}\left(7-6 \frac{r^{2}}{\left\langle r^{2}\right\rangle_{0}}+\frac{r^{4}}{\left\langle r^{2}\right\rangle_{0}^{2}}\right)\left(3 x^{2}-r^{2}\right) /\left\langle r^{2}\right\rangle_{0} \\
\alpha_{4} \equiv 3^{4}\left(\frac{77}{3}-33 \frac{r^{2}}{\left\langle r^{2}\right\rangle_{0}}+11 \frac{r^{4}}{\left\langle r^{2}\right\rangle_{0}^{2}}-\frac{r^{6}}{\left\langle r^{2}\right\rangle_{0}^{3}}\right)\left(3 x^{2}-r^{2}\right) /\left\langle r^{2}\right\rangle_{0}
\end{gathered}
$$

For $\left(\overline{\cos ^{2} \theta_{y}}\right)_{\mathbf{r}}$ the variable $x$ is replaced by $y$. The parameters $\beta_{i}$ are functions of the end-to-end vector magnitude only and therefore are the same for $x$ and $y$ components:

$$
\begin{gathered}
\beta_{1} \equiv 15-30 \frac{r^{2}}{\left\langle r^{2}\right\rangle_{0}}+9 \frac{r^{4}}{\left\langle r^{2}\right\rangle_{0}^{2}} \\
\beta_{2} \equiv 105-315 \frac{r^{2}}{\left\langle r^{2}\right\rangle_{0}}+189 \frac{r^{4}}{\left\langle r^{2}\right\rangle_{0}^{2}}-27 \frac{r^{6}}{\left\langle r^{2}\right\rangle_{0}^{3}} \\
\beta_{3} \equiv 945-3780 \frac{r^{2}}{\left\langle r^{2}\right\rangle_{0}}+3402 \frac{r^{4}}{\left\langle r^{2}\right\rangle_{0}^{2}}-972 \frac{r^{6}}{\left\langle r^{2}\right\rangle_{0}^{3}}+ \\
81 \frac{r^{8}}{\left\langle r^{2}\right\rangle_{0}^{4}}
\end{gathered}
$$

\section{References and Notes}

(1) Nagai, K. J. Chem. Phys. 1964, 40, 2818.
(2) Flory, P. J. Statistical Mechanics of Chain Molecules; Interscience: New York, 1969.

(3) Mark, J. E.; Erman, B. Rubberlike Elasticity. A Molecular Primer; Wiley-Interscience: New York, 1988; pp 196.

(4) Erman, B.; Haliloglu, T.; Bahar, I.; Mark, J. E. Macromolecules 1991, 24, 901.

(5) Fraser, R. D. B. J. Chem. Phys. 1953, 21, 1511.

(6) Read, B. E.; Stein, R. S. Macromolecules 1968, 1, 116.

(7) Treloar, L. R. G. The Physics of Rubber Elasticity; Clarendon Press; 1975

(8) Jarry, J. P.; Monnerie, L. Macromolecules 1979, 12, 316.

(9) Deloche, B.; Samulski, E. T. Macromolecules 1981, 14, 575.

(10) Deloche, B.; Dubault, A.; Herz, J.; Lapp, A. Europhys. Lett. 1986, 1,629

(11) Dubault, A.; Deloche, B.; Herz, J. Polymer 1984, 25, 1405.

(12) Dubault, A.; Deloche, B.; Herz, J. Macromolecules 1987, 20 , 2096.

(13) Besbes, S.; Cermelli, I.; Bokobza, L.; Monnerie, L.; Bahar, I.; Erman, B.; Herz, J. Macromolecules 1992, 25, 1949.

(14) Besbes, S.; Bokobza, L.; Monnerie, L.; Bahar, I.; Erman, B. Polymer 1993, 34, 1179.

(15) Kanberoğlu, C.; Bahar, I.; Erman, B. Polymer 1993, 34, 4997.

(16) Mitchell, G. R. Polymer 1984, 25, 1562.

(17) Mitchell, G. R. Br. Polymer J. 1985, 17, 111.

(18) Kuhn, W.; Grün, F. Kolloid-Z. 1942, 101, 248.

(19) Roe, R.; Krigbaum, W. R. J. Appl. Phys. 1964, 35, 2215.

(20) Haliloglu, T.; Bahar, I.; Erman, B. Comput. Polym. Sci. 1991, 1,151 .

(21) Lapersonne, P.; Tassin, J. F.; Sergot, P.; Monnerie, L.; Le Bourvellec, G. Polymer 1989, 30, 1558.

(22) Queslel, J. P.; Erman, B.; Monnerie, L. Macromolecules 1985, 18, 1991.

(23) Jarvis, D. A.; Hutchinson, I. J.; Bower, D. I.; Ward, I. M. Polymer 1980, 21, 41.

(24) Zhao, Y.; Jasse, B.; Monnerie, L. Polymer 1991, 32, 209.

(25) Cakmak, M.; White, J. L.; Spruiell, J. E. J. Polym. Eng. Sci. $1990,6,291$.

(26) Erman, B.; Bahar, I. Macromolecules 1988, 21, 452.

(27) Flory, P. J.; Yoon, D. Y. J. Chem. Phys. 1974, 61, 5358.

(28) Yoon, D. Y.; Flory, P. J. J. Chem. Phys. 1974, 61, 5366

(29) Flory, P. J. Macromolecules 1974, 7, 381.

(30) Erman, B.; Monnerie, L. Macromolecules 1985, 26, 167.

(31) Erman, B.; Bahar, I.; Kloczkowski, A.; Mark, J. E. Macromolecules $1990,23,5335$.

(32) Sotta, P.; Deloche, B.; Herz, J.; Lapp, A.; Durand, D.; Rabadeux, J.-C. Macromolecules 1987, 20, 2769.

MA941013Y 